\title{
Tests of direct and indirect CPT violation at a $B$ factory
}

\author{
Don Colladay, V. Alan Kostelecký \\ Physics Department, Indiana University, Bloomington, IN 47405, USA \\ Received 7 November 1994 \\ Editor: H. Georgi
}

\begin{abstract}
The issue of testing CPT invariance at a $B$ factory is considered. We present asymmetries that permit a clean extraction of quantities parametrizing direct and indirect CPT violation, using information from $\Upsilon(4 S)$ decay via coherent $B_{d} \overline{B_{d}}$ pairs into various final states. Estimates are given of the bounds on CPT violation that could be obtained in present and planned machines.
\end{abstract}

\section{Introduction}

Invariance under the combined discrete symmetry CPT is known to be a feature of local relativistic pointparticle field theories [1-7], including the standard model. The current bound on CPT violation is obtained from experiments in the kaon system [8,9], where one figure of merit is a few parts in $10^{18}$. The basic reason underlying the feasibility of high precision tests of CPT is the interferometric nature of the kaon system, in which a small mass difference and the possibility of strangeness oscillations amplify effects that would otherwise be unobservable.

The $B^{0}-\overline{B^{0}}$ system is also an interferometric system, but one with detailed properties different from the kaon system. In addition to its use as a probe of standard-model features and parameters, it is also possible at least in principle to use a $B$ factory to further test CPT invariance. At present, several efforts are underway to develop $B$ factories, for example, at Cornell and SLAC in the US and at KEK in Japan. ${ }^{1}$ These

\footnotetext{
${ }^{1}$ A discussion of some of the many extant proposals can be found in Ref. [10].
}

machines are designed to create relatively high fluxes of $\curlyvee(4 S)$ and hence of correlated $B_{d}^{0}-\overline{B_{d}^{0}}$ pairs.

Other than intrinsic interest, additional motivation for testing CPT comes from the possibility that it is violated in the context of string theory, ultimately due to the nonlocal nature of strings [11]. It is natural to expect any effects of this type to be strongly suppressed at accessible energy scales. However, the interferometric sensitivity of neutral-meson systems may make such effects observable [12,13]. In any neutral-meson system, the string scenario suggests that direct CPT violation would be too small to measure, so all complex direct-violation parameters are effectively zero. In contrast, for each meson system the real and imaginary parts of the complex indirect-violation parameter satisfy a certain condition (see Eq. (2) below), while the magnitude of this parameter can in principle take values attainable in the present or next generation of machines.

Experimentally testing these ideas evidently requires isolating the various quantities parametrizing CPT violation. In the decay of a vector meson to a correlated neutral-meson pair, a CP-violating effect 
(together with the associated $\mathrm{T}$ or CPT violation) can be extracted through consideration of selected asymmetries in decay rates to various final states. For the $B$ system, the literature contains some asymmetries that vanish if CPT is preserved [13-15]. However, the issue of a clean extraction of CPT-violation parameters has been an open problem because $\mathrm{T}$ and $\mathrm{CPT}$ effects have not yet been disentangled. One purpose of this paper is to fill this gap. We present a means of separating quantities parametrizing both direct and indirect CPT violation by identifying certain asymmetries that isolate these parameters. We also obtain estimates for the bounds on CPT violation that could be obtained in present and planned $B$ factories using these asymmetries.

\section{Preliminaries}

In this section, we present key definitions and equations used later in the paper. Generally, our conventions [13] are analogues of standard ones in the kaon system. Throughout, we take all CP violation (and hence $\mathrm{T}$ and CPT violation) to be small, and we neglect terms that are higher-order in small quantities.

The eigenvectors of the effective hamiltonian for the $B^{0}-\bar{B}^{0}$ system are given by

$$
\begin{aligned}
\left|B_{S}\right\rangle & =\frac{1}{\sqrt{2}}\left[\left(1+\epsilon_{B}+\delta_{B}\right)\left|B^{0}\right\rangle\right. \\
+ & \left.\left(1-\epsilon_{B}-\delta_{B}\right)\left|\overline{B^{0}}\right\rangle\right], \\
\left|B_{L}\right\rangle & =\frac{1}{\sqrt{2}}\left[\left(1+\epsilon_{B}-\delta_{B}\right)\left|B^{0}\right\rangle\right. \\
& \left.-\left(1-\epsilon_{B}+\delta_{B}\right)\left|\overline{B^{0}}\right\rangle\right] .
\end{aligned}
$$

The CP-viulating complex parameters $\epsilon_{B}$ and $\delta_{B}$ are measures of indirect $\mathrm{T}$ and indirect CPT violation, respectively. For completeness, we note here that in the string-inspired scenario for CPT violation the quantity $\delta_{B}$ satisfies

$$
\operatorname{Im} \delta_{B}= \pm \frac{\Delta \gamma}{2 \Delta m} \operatorname{Re} \delta_{B},
$$

where $\Delta \gamma=\gamma_{S}-\gamma_{L}$ and $\Delta m=m_{L}-m_{S}$ denote lifetime and mass differences, respectively, of the physical particles $B_{S}$ and $B_{L}$. Except where stated, we do not impose the condition (2) in what follows.
The initial state $|i\rangle$ of the $B_{d}-\overline{B_{d}}$ pair arising from the $Y(4 S)$ decay has $J^{\mathrm{PC}}=1^{--}$. Taking the direction of the $B$-meson momenta to be along the $z$ axis, this state can be written as

$$
\begin{aligned}
|\hat{i}\rangle & =\frac{1}{\sqrt{2}}\left[\left|B_{S}(\hat{z}) B_{L}(-\hat{z})\right\rangle\right. \\
& \left.-\left|B_{L}(\hat{z}) B_{S}(-\hat{z})\right\rangle\right],
\end{aligned}
$$

where the argument $( \pm \hat{z})$ denotes a meson moving in the $\pm \hat{z}$ direction. In what follows, we label the two mesons by $\alpha=1,2$ and take them to decay into final states $\left|f_{\alpha}\right\rangle$ at times $t_{\alpha}$ as measured in the rest frame of the $Y(4 S)$ decay. Defining the transition amplitudes

$a_{\alpha S}=\left\langle f_{\alpha}|T| B_{S}\right\rangle, \quad a_{\alpha L}=\left\langle f_{\alpha}|T| B_{L}\right\rangle$

and their ratios $\eta_{\alpha}=a_{\alpha L} / a_{\alpha S}$, it follows that the amplitude $\mathcal{A}_{12}\left(t_{1}, t_{2}\right)$ for the decay is

$$
\begin{aligned}
& \mathcal{A}_{12}\left(t_{1}, t_{2}\right)=\frac{1}{\sqrt{2}} a_{1 S} a_{2 S} \\
& \quad \times\left(\eta_{2} \exp \left[-i\left(m_{S} t_{1}+m_{L} t_{2}\right)-\frac{1}{2}\left(\gamma_{S} t_{1}+\gamma_{L} t_{2}\right)\right]\right. \\
& \left.-\eta_{1} \exp \left[-i\left(m_{L} t_{1}+m_{S} t_{2}\right)-\frac{1}{2}\left(\gamma_{L} t_{1}+\gamma_{S} t_{2}\right)\right]\right) .
\end{aligned}
$$

Experiments observe integrated rates. It is useful to introduce first the once-integrated decay rate

$I\left(f_{1}, f_{2}, \pm v\right)=\frac{1}{2} \int_{v}^{\infty} d t\left|\mathcal{A}_{12}\left(t_{1}, t_{2}\right)\right|^{2}$,

where $t=t_{1}+t_{2}$ is the sum of the decay times and $v=\left|t_{2}-t_{1}\right|$ is the magnitude of their difference. Calculation gives [13]

$$
\begin{aligned}
& I\left(f_{1}, f_{2}, \pm v\right)=\frac{\left|a_{1 S} a_{2 S} \eta_{1}\right|^{2}}{2 \gamma} e^{-\frac{1}{2} \gamma v} \\
& \times\left[e^{\mp \frac{1}{2} \Delta \gamma v}+\left|r_{21}\right|^{2} e^{ \pm \frac{1}{2} \Delta \gamma v}\right. \\
& \left.-2\left|r_{21}\right| \cos (\Delta m v \pm \Delta \phi)\right],
\end{aligned}
$$

where $\gamma=\gamma_{S}+\gamma_{L}, r_{21}=\eta_{2} / \eta_{1}$, and $\Delta \phi=\phi_{1}-\phi_{2}$, with $\phi_{\alpha}$ given by $\eta_{\alpha} \equiv\left|\eta_{\alpha}\right| e^{i \phi_{\alpha}}$. It is also useful to define symbols for two frequently occurring combinations of the basic parameters $\Delta m, \gamma$, and $\Delta \gamma$ :

$a^{2}=\Delta m^{2}+\frac{1}{4} \Delta \gamma^{2}$,

$b^{2}=\Delta m^{2}+\frac{1}{4} \gamma^{2}$. 
In subsequent sections, we use the following twiceintegrated decay rates:

$$
\begin{aligned}
& \Gamma\left(f_{1}, f_{2}\right)=\int_{-\infty}^{\infty} d v I\left(f_{1}, f_{2}, v\right) \\
& =\frac{1}{2 \gamma_{S} \gamma_{L}}\left[\left|a_{1 S} a_{2 L}\right|^{2}+\left|a_{1 L} a_{2 S}\right|^{2}\right. \\
& \left.-\frac{\gamma_{S} \gamma_{L}}{b^{2}}\left(a_{1 S}^{*} a_{2 L}^{*} a_{1 L} a_{2 S}+\text { c.c. }\right)\right] \text {, } \\
& \Gamma^{+}\left(f_{1}, f_{2}\right)=\int_{0}^{\infty} d v I\left(f_{1}, f_{2}, v\right) \\
& =\frac{1}{2 \gamma}\left[\frac{\left|a_{1 s} a_{2 L}\right|^{2}}{\gamma_{L}}+\frac{\left|a_{1 L} a_{2 S}\right|^{2}}{\gamma_{S}}\right. \\
& \left.-\left(\frac{a_{1 S}^{*} a_{2 L}^{*} a_{1 L} a_{2 S}}{\frac{1}{2} \gamma-i \Delta m}+\text { c.c. }\right)\right] \text {, } \\
& \Gamma^{-}\left(f_{1}, f_{2}\right)=\int_{-\infty}^{0} d v I\left(f_{1}, f_{2}, v\right) \\
& =\frac{1}{2 \gamma}\left[\frac{\left|a_{1 s} a_{2 L}\right|^{2}}{\gamma_{S}}+\frac{\left|a_{1 L} a_{2 S}\right|^{2}}{\gamma_{L}}\right. \\
& \left.-\left(\frac{a_{1 S}^{*} a_{2 L}^{*} a_{1 L} a_{2 S}}{\frac{1}{2} \gamma+i \Delta m}+\text { c.c. }\right)\right] \text {, } \\
& \Gamma_{\text {incl }}^{+}\left(f_{1}\right)=\sum_{f_{2}} \Gamma^{+}\left(f_{1}, f_{2}\right) \\
& =\frac{1}{2 \gamma}\left[\left|a_{1 s}\right|^{2}+\left|a_{1 L}\right|^{2}\right. \\
& -2\left[a_{1 S}^{*} a_{1 L}\left(\operatorname{Re} \epsilon_{B}+i \operatorname{Im} \delta_{B}\right)+\text { c.c. }\right] \text {. }
\end{aligned}
$$

Note that we do not need the quantity $\Gamma_{\text {incl }}^{-}\left(f_{1}\right) \equiv$ $\sum_{f_{2}} \Gamma^{-}\left(f_{1}, f_{2}\right) \neq \Gamma_{\text {incl }}^{+}\left(f_{1}\right)$ in what follows.

\section{Direct CPT violation}

In this section we consider certain special $B$ decays, called semileptonic-type decays, for each of which we present an asymmetry that extracts a corresponding parameter measuring direct CPT violation. These decays include the usual semileptonic decays, along with a special class of other modes $B^{0} \rightarrow f$ for which there is no lowest-order weak process that would allow a significant contamination of either $\overline{B^{0}} \rightarrow f$ or $B^{0} \rightarrow \bar{f}$. Among the decays observed to date [16], the semileptonic-type ones include $B^{0} \rightarrow$ $D^{-} D_{s}^{+}, B^{0} \rightarrow J / \psi K^{+} \pi^{-}, B^{0} \rightarrow J / \psi K^{* 0}(892)$, $B^{0} \rightarrow \psi(2 S) K^{* 0}(892)$, and similar decays into excited states. Note that the mode $B^{0} \rightarrow J / \psi K^{0}$ is excluded because the $K^{0}$ is not a directly observable final state. For the other modes that have been seen, a CKM-suppressed process exists contributing to the contaminating transitions.

The various transition amplitudes associated with the decay of the neutral $B$ meson to a semileptonictype final state $f$ can be parametrized as follows $[17,18]$ :

$$
\begin{aligned}
& \left\langle f|T| B^{0}\right\rangle=F_{f}\left(1-y_{f}\right), \\
& \left\langle f|T| \overline{B^{0}}\right\rangle=x_{f} F_{f}\left(1-y_{f}\right), \\
& \left\langle\bar{f}|T| \overline{B^{0}}\right\rangle=F_{f}^{*}\left(1+y_{f}^{*}\right), \\
& \left\langle\bar{f}|T| B^{0}\right\rangle=\bar{x}_{f}^{*} F_{f}^{*}\left(1+y_{f}^{*}\right) .
\end{aligned}
$$

In these expressions, the parameters on the right-hand side are all complex. The independent complex quantities $x_{f}$ and $\bar{x}_{f}$ are included to allow for the possibility of a violation in the $\Delta B=\Delta Q$ rule. They vanish if the rule is exact, so in what follows we treat them as small. If T invariance holds, all the quantities $x_{f}, \bar{x}_{f}$, $F_{f}$, and $y_{f}$ are real. If CPT invariance holds, $x_{f}=\bar{x}_{f}$ and $y_{f}=0$. The parameter $y_{f}$ is therefore a measure of direct CPT violation in the decay to the final state $f$. Its real part $\operatorname{Re} y_{f}$ is the present focus of our attention.

Each individual final state $f$ offers the opportunity to test direct CPT violation through a measurement of the corresponding $\operatorname{Re} y_{f}$. To extract $\operatorname{Re} y_{f}$ from rate information, we first determine the amplitudes introduced in Eq. (4). Using the above definitions, to first order in small quantities we find

$$
\begin{aligned}
& a_{f S}=\frac{1}{\sqrt{2}} F_{f}\left(1+\epsilon_{B}+\delta_{B}-y_{f}+x_{f}\right), \\
& a_{f L}=\frac{1}{\sqrt{2}} F_{f}\left(1+\epsilon_{B}-\delta_{B}-y_{f}-x_{f}\right), \\
& a_{\bar{f} S}=\frac{1}{\sqrt{2}} F_{f}^{*}\left(1-\epsilon_{B}-\delta_{B}+y_{f}^{*}+\bar{x}_{f}^{*}\right), \\
& a_{\bar{f} L}=-\frac{1}{\sqrt{2}} F_{f}^{*}\left(1-\epsilon_{B}+\delta_{B}+y_{f}^{*}-\bar{x}_{f}^{*}\right) .
\end{aligned}
$$


With these amplitudes, we can calculate for the correlated $B$ pairs the inclusive decay rates $\Gamma_{\text {incl }}^{+}(f)$ of the type in Eq. (12), where one final state is required to be $f$ while the other is arbitrary and where the decay into $f$ occurs first. We can then extract the asymmetry $A_{f}^{+}$between decays into $f$ and $\bar{f}$. This asymmetry is proportional to $\operatorname{Re} y_{f}$.

Explicit calculation gives ${ }^{2}$

$$
\begin{aligned}
A_{f}^{+} & \equiv \frac{\Gamma_{\text {incl }}^{+}(f)-\Gamma_{\text {incl }}^{+}(\bar{f})}{\Gamma_{\text {incl }}^{+}(f)+\Gamma_{\text {incl }}^{+}(\bar{f})} \\
& =-2 \operatorname{Re} y_{f} .
\end{aligned}
$$

This asymmetry provides a clean way of extracting an effect from direct CPT violation, independently of any $\mathrm{T}$ or indirect CPT violation. In Section 5 below, we comment on the experimental feasibility of using this asymmetry and we provide an estimate of the bound attainable on $\operatorname{Re} y_{f}$ using Eq. (15).

Although not central to the purpose of the present paper it is worth noting that, once a bound (or value) on $\operatorname{Re} y_{f}$ has been extracted, the quantity $\operatorname{Re} \epsilon_{B}$ measuring $\mathrm{T}$ violation can be determined without making the assumption of CPT invariance. Consider the total integrated rate asymmetry $\Lambda_{f, \bar{f}}^{\text {tot }}$, given by

$$
\begin{aligned}
A_{f, \bar{f}}^{\mathrm{tot}} & \equiv \frac{\Gamma(f, f)-\Gamma(\bar{f}, \bar{f})}{\Gamma(f, f)+\Gamma(\bar{f}, \bar{f})} \\
& =4 \operatorname{Re}\left(\epsilon_{B}-y_{f}\right) .
\end{aligned}
$$

We sec that a nonzero value of the combination $\frac{1}{4}\left(A_{f, \bar{f}}^{\text {tot }}-2 A_{f}^{+}\right) \equiv \operatorname{Re} \epsilon_{B}$ for any given final state $f$ of the semileptonic type can be unambiguously attributed to the T-violation parameter $\operatorname{Re} \epsilon_{B}$ without making the assumption of CPT invariance.

We also note that the derivation of Eqs. (15) and (16) applies also to the $K^{0}-\overline{K^{0}}$ system, when the final state is $f=\pi^{-} l^{+} \nu_{l}$. The quantities $\operatorname{Re} y_{l}$ and $\operatorname{Re} \epsilon_{K}$ for this system can therefore also be obtained in this way. Indeed, one can show that, in the absence of CPT invariance and without resorting to a fit to a $\Delta t$ dependent quantity, this method is the only way to extract $\operatorname{Re} \epsilon_{K}$ from integrated asymmetries in $\phi$ decay.

\footnotetext{
${ }^{2}$ An analysis keeping terms to all orders in $x_{f}$ and $\bar{x}_{f}$ shows that Eq. (15) has no linear corrcctions in thesc quantitics.
}

\section{Indirect CPT violation}

The complex parameter $\delta_{B}$ is a measure of indirect CPT violation. We first consider a means of obtaining its real part and subsequently address the issue of the imaginary part.

Consider decays of the correlated $B$ pair into either $J / \psi K_{S}$ or $J / \psi K_{L}$ in one decay channel and a semileptonic-type state $f$ in the other. In analogy with Eq. (14), we define the transition amplitudes to the states involving $K^{0}$ and $\overline{K^{0}}$ as follows:

$$
\begin{aligned}
& \left\langle J / \psi K^{0}|T| B^{0}\right\rangle=F_{J / \psi}\left(1-y_{J / \psi}\right), \\
& \left\langle J / \psi K^{0}|T| \overline{B^{0}}\right\rangle=x_{J / \psi} F_{J / \psi}\left(1-y_{J / \psi}\right), \\
& \left\langle J / \psi \overline{K^{0}}|T| \overline{B^{0}}\right\rangle=F_{J / \psi}^{*}\left(1+y_{J / \psi}^{*}\right), \\
& \left\langle J / \psi \overline{K^{0}}|T| B^{0}\right\rangle=\bar{x}_{J / \psi}^{*} F_{J / \psi}^{*}\left(1+y_{J / \psi}^{*}\right) .
\end{aligned}
$$

As before, this allows for possible violation of the $\Delta B=\Delta Q$ rule via $x_{J / \psi}$ and $\bar{x}_{J / \psi}$, while the complex parameter $y_{J / \psi}$ characterizes direct CPT violation. These parameters are assumed small in what follows.

The final products of the $Y(4 S)$ decay involve $K_{S}$ and $K_{L}$ rather than $K^{0}$ and $\overline{K^{0}}$. The ratios of matrix elements useful for asymmetry determination therefore involve the former states. Using the definitions (17), we obtain:

$$
\begin{aligned}
& \eta_{J / \psi K_{S}} \equiv \frac{\left\langle J / \psi K_{S}|T| B_{L}\right\rangle}{\left\langle J / \psi K_{S}|T| B_{S}\right\rangle} \\
& =\epsilon_{K}^{*}+\epsilon_{B}+\delta_{K}^{*}-\delta_{B} \\
& \quad-\frac{\operatorname{Re}\left(F_{J / \psi} y_{J / \psi}\right)}{\operatorname{Re} F_{J / \psi}}-\frac{1}{2 \operatorname{Re} F_{J / \psi}}\left(x_{J / \psi} F_{J / \psi}-\bar{x}_{J / \psi}^{*} F_{J / \psi}^{*}\right) \\
& \quad+i \frac{\operatorname{Im} F_{J / \psi}}{\operatorname{Re} F_{J / \psi}}\left[1-i \frac{\operatorname{Im} F_{J / \psi}}{\operatorname{Re} F_{J / \psi}}\left(\epsilon_{K}^{*}+\epsilon_{B}+\delta_{K}^{*}+\delta_{B}\right)\right. \\
& \quad-\frac{1}{2 \operatorname{Re} F_{J / \psi}}\left(x_{J / \psi} F_{J / \psi}+\bar{x}_{J / \psi}^{*} F_{J / \psi}^{*}\right) \\
& \left.\quad+i \frac{\operatorname{Im}\left(F_{J / \psi} y_{J / \psi}\right)}{\operatorname{Re} F_{J / \psi}}\right],
\end{aligned}
$$




$$
\begin{aligned}
\eta_{J / \psi K_{L}} \equiv \frac{\left\langle J / \psi K_{L}|T| B_{S}\right\rangle}{\left\langle J / \psi K_{L}|T| B_{L}\right\rangle} \\
=\epsilon_{K}^{*}+\epsilon_{B}-\delta_{K}^{*}+\delta_{B} \\
-\frac{\operatorname{Re}\left(F_{J / \psi} y_{J / \psi}\right)}{\operatorname{Re} F_{J / \psi}}+\frac{1}{2 \operatorname{Re} F_{J / \psi}}\left(x_{J / \psi} F_{J / \psi}-\bar{x}_{J / \psi}^{*} F_{J / \psi}^{*}\right) \\
+i \frac{\operatorname{Im} F_{J / \psi}}{\operatorname{Re} F_{J / \psi}}\left[1-i \frac{\operatorname{Im} F_{J / \psi}}{\operatorname{Re} F_{J / \psi}}\left(\epsilon_{K}^{*}+\epsilon_{B}-\delta_{K}^{*}-\delta_{B}\right)\right. \\
+\frac{1}{2 \operatorname{Re} F_{J / \psi}}\left(x_{J / \psi} F_{J / \psi}+\bar{x}_{J / \psi}^{*} F_{J / \psi}^{*}\right) \\
\left.+i \frac{\operatorname{Im}\left(F_{J / \psi} y_{J / \psi}\right)}{\operatorname{Re} F_{J / \psi}}\right] .
\end{aligned}
$$

In these equations, the parameters $\epsilon_{K}$ and $\delta_{K}$ are quantities parametrizing indirect $\mathrm{T}$ and CPT violation in the kaon system.

The goal is to identify an asymmetry or combination of asymmetries permitting the extraction of $\operatorname{Re} \delta_{B}$. To this end, we introduce the following two rate asymmetries:

$$
\begin{gathered}
A_{f, K_{S}} \equiv \frac{\Gamma\left(f, J / \psi K_{S}\right)-\Gamma\left(\bar{f}, J / \psi K_{S}\right)}{\Gamma\left(f, J / \psi K_{S}\right)+\Gamma\left(\bar{f}, J / \psi K_{S}\right)} \\
=2 \operatorname{Re}\left(\epsilon_{B}-y_{f}-\delta_{B}\right) \\
-\frac{2 \gamma_{S} \gamma_{L}}{b^{2}} \operatorname{Re}\left(\eta_{J / \psi K_{S}}\right),
\end{gathered}
$$

and

$$
\begin{aligned}
A_{f, K_{L}} & \equiv \frac{\Gamma\left(f, J / \psi K_{L}\right)-\Gamma\left(\bar{f}, J / \psi K_{L}\right)}{\Gamma\left(f, J / \psi K_{L}\right)+\Gamma\left(\bar{f}, J / \psi K_{L}\right)} \\
& =2 \operatorname{Re}\left(\epsilon_{B}-y_{f}+\delta_{B}\right) \\
& -\frac{2 \gamma_{S} \gamma_{L}}{b^{2}} \operatorname{Re}\left(\eta_{J / \psi} K_{L}\right) .
\end{aligned}
$$

In deriving the explicit form of these two asymmetries, we have assumed that violations of the $\Delta B=\Delta Q$ rule are independent of violations of CPT invariance, so that $x_{f}=\bar{x}_{f}$ and $x_{J / \psi}=\bar{x}_{J / \psi}$. Since $\operatorname{Im} F_{J / \psi}$ controls the direct $T$ violation in these processes, we have also treated it as a small quantity.

The difference between the asymmetries in Eqs. (19) and (20) is a function of CPT-violating parameters: ${ }^{3}$

\footnotetext{
${ }^{3} \mathrm{~A}$ derivation relaxing the constraint of small direct $\mathrm{T}$ violation shows that Eq. (21) is correct up to terms simultaneously quadratic in $\operatorname{Im} F_{J / \psi}$ and linear in $\delta_{B}$ or $\delta_{K}$.
}

$$
\begin{aligned}
A_{L, S} & \equiv A_{f, K_{L}}-A_{f, K_{S}} \\
& =\frac{4}{b^{2}}\left[a^{2} \operatorname{Re} \delta_{B}+\gamma_{S} \gamma_{L} \operatorname{Re} \delta_{K}\right] .
\end{aligned}
$$

The measurement of $A_{L, S}$ provides a means of obtaining a fairly stringent bound on $\operatorname{Re} \delta_{B}$. The point is that the parameter $\operatorname{Re} \delta_{K}$ can be bounded using rate information from semileptonic $K$ decays. This is discussed further in the next section. Note also that this result is independent of the state $f$, which makes the statistics more favorable by allowing a sum over the class of semileptonic-type final states. We emphasize that the asymmetry combination $A_{L, S}$ permits the extraction of $\operatorname{Re} \delta_{B}$ independently of effects from direct CPT violation and direct or indirect $\mathrm{T}$ violation arising in either the $B$ or the $K$ systems.

Once a bound on $\operatorname{Re} \delta_{B}$ is established, $\operatorname{Im} \delta_{B}$ can in turn be obtained from a measurement of double semileptonic decay rates of the $Y(4 S)$. The quantity to be measured is [13]

$$
\begin{aligned}
A_{f, \bar{f}} & \equiv \frac{\Gamma^{+}(f, \bar{f})-\Gamma^{-}(f, \bar{f})}{\Gamma^{+}(f, \bar{f})+\Gamma^{-}(f, \bar{f})} \\
& =4 \frac{b^{2} \Delta \gamma \operatorname{Re} \delta_{B}+2 \Delta m \gamma_{s} \gamma_{L} \operatorname{Im} \delta_{B}}{\gamma\left(b^{2}+\gamma_{S} \gamma_{L}\right)}
\end{aligned}
$$

In obtaining the explicit form of this asymmetry, it is assumed that any violation of the $\Delta B=\Delta Q$ rule is independent of CP violation, so that $x_{f}=\bar{x}_{f}^{*}$.

\section{Estimates of bounds attainable}

In this section, we investigate the bounds attainable from the above analyses on the quantities parametrizing direct and indirect CPT violation in the $B$ system. Following the methods of Ref. [19], for each of the relevant quantities we provide an estimate of the number of $Y(4 S)$ events required to reduce the error in the associated asymmetry to one standard deviation.

In general, for an asymmetry $A=\left(N_{+}-\right.$ $\left.N_{-}\right) /\left(N_{+}+N_{-}\right)$, the binomial distribution implies that the expected number of events $\left\langle N_{+}\right\rangle$required to observe a nonzero $\langle A\rangle$ at the $N \sigma$ level is $N^{2}(1+\langle A\rangle)\left(1-\langle A\rangle^{2}\right) / 2\langle A\rangle^{2}$. To convert this to $Y(4 S)$ events, this number must be multiplied by two to account for the branching ratio of $Y(4 S)$ into two ncutral $B$ mesons and by the inverse branching ratio 
for the latter into the relevant final states. The assumption that any $\mathrm{T}$ and $\mathrm{CPT}$ violations are small implies that interference effects in the correlated decays can be neglected.

We first consider bounds on the various $\operatorname{Re} y_{f}$, which provide measures of direct CPT violation. The relevant asymmetry is $A_{f}^{+}$, given by Eq. (15). Since the second final state is unrestricted, it is sufficient to multiply only by the inverse branching ratio for the process $B^{0} \rightarrow f$. An additional multiplicative factor appears because the asymmetry involves only those events for which the decay into $f$ occurs first. This factor is two because in the $B$ system $\gamma_{S} \approx \gamma_{L}$, which makes $\Delta t>0$ events about as likely as $\Delta t<0$ events. Combining this information, we find that the number $N_{\Upsilon(4 S)}\left(\operatorname{Re} y_{f}\right)$ of $\Upsilon(4 S)$ events needed to reduce the error in $\operatorname{Re} y_{f}$ to within one standard deviation $\sigma$ is

$N_{\Upsilon(4 S)}\left(\operatorname{Re} y_{f}\right) \simeq \frac{1}{2 \sigma^{2} \operatorname{BR}\left(B^{0} \rightarrow f\right)}$.

Next, we consider the bound on $\operatorname{Re} \delta_{B}$, parametrizing indirect CPT violation. For the combination $A_{L, S}$ of asymmetries given by Eq. (21), the errors in $A_{f, K_{S}}$ and $A_{f, K_{L}}$ must be combined in quadrature. Also, an estimate is needed of the size of the coefficients of $\operatorname{Re} \delta_{B}$ and $\operatorname{Re} \delta_{K}$ in the equation. For the latter, we take [16] $\gamma_{S} \approx \gamma_{L}$ and $^{4} x=2 \Delta m / \gamma \simeq \pm 0.71$. With these values, Eq. (21) becomes

$A_{L, S} \approx 1.3 \operatorname{Re} \delta_{B}+2.7 \operatorname{Re} \delta_{K}$.

Since $A_{L, S}$ is independent of the specific semileptonic-type final state $f$, the corresponding branching ratios can be summed. This gives $\sum_{f} \mathrm{BR}\left(B^{0} \rightarrow f\right) \simeq$ $15 \%$. Since the $K^{0}$ is roughly $50 \% K_{S}$ and $50 \% K_{L}$, we take [16]

$$
\begin{aligned}
& \mathrm{BR}\left(B^{0} \rightarrow J / \psi K_{S}\right) \approx \mathrm{BR}\left(B^{0} \rightarrow J / \psi K_{L}\right) \\
& \quad \approx \frac{1}{2} \mathrm{BR}\left(B^{0} \rightarrow J / \psi K^{0}\right) \\
& \simeq 3.8 \times 10^{-4} .
\end{aligned}
$$

From the limit cited in Ref. [20], the current bound on $\operatorname{Re} \delta_{K}$ lies at the $10^{-3}$ level. For simplicity, take $\operatorname{Re} \delta_{K}$ to be zero, i.e., sufficiently well bounded by $K$-decay

\footnotetext{
${ }^{4}$ The value of $x$ quoted is a lower bound, $|x| \geq 0.71$, if CPT invariance is not assumed [14]. However, a value above this bound improves the statistics obtained below.
}

experiments. We also take the errors in the asymmetries $A_{f, K_{S}}$ and $A_{f, K_{L}}$ to be roughly equal. Then, we find that the number $N_{\Upsilon(4 S)}\left(\operatorname{Re} \delta_{B}\right)$ of $Y(4 S)$ events needed to reduce the error in $\operatorname{Re} \delta_{B}$ to within one standard deviation $\sigma$ is

$N_{Y(4 S)}\left(\operatorname{Re} \delta_{B}\right) \simeq \frac{1.8 \times 10^{4}}{\sigma^{2}}$

Finally, given a bound on $\operatorname{Re} \delta_{B}$, Eq. (22) can be used to provide an estimate of the number $N_{Y(4 S)}\left(\operatorname{Im} \delta_{B}\right)$ of $Y(4 S)$ events needed to reduce the error in $\operatorname{Im} \delta_{B}$ to within one standard deviation $\sigma$. For example, if the string-inspired relation (2) is valid, a similar calculation to those above gives [13]

$N_{Y(4 S)}\left(\operatorname{Im} \delta_{B}\right) \simeq \frac{5}{\sigma^{2}}$

The ease with which experimental information can be obtained differs for the above quantities. In particular, the asymmetries involved in bounding $\operatorname{Re} y_{f}$ and $\operatorname{Im} \delta_{B}$ require knowledge of the sign of $\Delta t$. However, at a symmetric $B$ factory the distance between decay vertices of the two $B$ mesons is only about $60 \mu \mathrm{m}$, and information about the location of the $Y(4 S)$ decay is difficult to acquire. A symmetric $B$ factory is therefore best suited to measure or bound $\operatorname{Re} \delta_{B}$. The situation is improved at an asymmetric $B$ factory, where the boost alters the topology of the events (see, e.g., Ref. [21].) This creates a greatly decreased angular separation and hence an easier determination of the sign of $\Delta t$.

\section{Summary}

We have presented asymmetries that allow the independent extraction of quantities parametrizing both direct and indirect CPT violation in the $B$ system. These asymmetries are given in Eq. (15) for $\operatorname{Re} y_{f}$, in Eq. (21) for $\operatorname{Re} \delta_{B}$, and in Eq. (22) for $\operatorname{Im} \delta_{B}$. We have also shown that, once direct violation is measured or bounded, the quantity $\operatorname{Re} \epsilon_{B}$ parametrizing indirect $T$ violation can be obtained from the asymmetry (16) without assumptions regarding CPT invariance.

Assuming no severe acceptance or background effects, it appears experimentally feasible to put bounds on both direct and indirect CPT violation. Estimates of the bounds attainable are given in Eq. (23) for $\operatorname{Re} y_{f}$, 
in Eq. (26) for $\operatorname{Re} \delta_{B}$, and in Eq. (27) for $\operatorname{Im} \delta_{B}$. Bounding $\operatorname{Re} \delta_{B}$ is possible at either a symmetric or an antisymmetric $B$ factory. This can be performed by comparing $B_{d}$ decays into $J / \psi K_{S}$ with decays into $J / \psi K_{L}$, without the need for information about $\Delta t$. Measurements of $\operatorname{Re} y_{f}$ and $\operatorname{Im} \delta_{B}$ require a knowledge of the sign of $\Delta t$, which is more easily obtained at an asymmetric factory. Accumulation of about $10^{7}$ or $10^{8}$ correlated $B_{d}-\overline{B_{d}}$ pairs, which could result from about one running ycar at a $B$ factory meeting typical design luminosities, should permit the determination of bounds on the various quantities to approximately the $10^{-2}$ level.

Independent examination of the different possible types of CPT violation in the $B$ system is worthwhile since CPT invariance is a fundamental symmetry of the standard model. If any violation is uncovered, the possibility of stringy effects in the system can be tested and the source can be isolated by the methods presented above.

\section{Acknowledgement}

This work was supported in part by the United States Department of Energy under grant number DE-FG0291ER40661.

\section{References}

[1] J. Schwinger, Phys. Rev. 82 (1951) 914.

[2] G. Lüders, Det. Kong. Danske Videnskabernes Selskab Mat.fysiske Meddelelser 28, No. 5 (1954); Ann. Phys. (N.Y.) 2 (1957) 1.
[3] J.S. Bell, Birmingham Univcrsity thesis (1954); Proc. Roy. Soc. (London) A 231 (1955) 479.

[4] W. Pauli, in: Niels Bohr and the Development of Physics, ed. W. Pauli, McGraw-Hill, New York, 1955, p. 30.

[5] G. Lüders and B. Zumino, Phys. Rev. 106 (1957) 385.

[6] R.F. Streater and A.S. Wightman, PCT, Spin and Statistics and All That, Benjamin Cummings, Reading, 1964.

[7] R. Jost, The General Theory of Quantized Fields, AMS, Providence, 1965.

[8] R. Carosi et al., Phys. Lett. B 237 (1990) 303.

[9] M. Karlsson et al., Phys. Rev. Lett. 64 (1990) 2976.

[10] P.E. Schlein, ed., Beauty '93, Nucl. Instr. Meth. A 333 (1993).

[11] V.A. Kostelecký and R. Potting, Nucl. Phys. B 359 (1991) 545.

[12] V.A. Kostelecký and R. Potting, in: Gamma Ray-Neutrino Cosmology and Planck Scale Physics, ed. D.B. Cline (World Scientific, Singapore, 1993).

[13] V.A. Kostelecký and R. Potting, Indiana University preprint IUHET 265, submitted for publication.

[14] M. Kobayashi and A.I. Sanda, Phys. Rev. Lett. 69 (1992) 3139.

[15] Zhi-zhong Xing, Phys. Rev. D 50 (1994) 2957.

[16] L. Montanet et al., Review of Particle Properties, Phys. Rev. D 50 (1994) 1173.

[17] T.D. Lee and C.S. Wu, Ann. Rev. Nucl. Sci. 16 (1966) 511.

[18] N.W. Tanner and R.H. Dalitz, Ann. Phys. 171 (1986) 463.

[19] I. Dunietz, J. Hauser and J.L. Rosner, Phys. Rev. D 35 (1987) 2166.

[20] T. Ruf, CPLEAR Collaboration, presented at the XXVII International Conference on High Energy Physics, Glasgow, Scotland, 20-27 July 1994.

[21] K.R. Schubert, Nucl. Phys. A 546 (1992) 337c. 